\title{
BMJ Open Constructing treatment episodes from concomitant medication logs: a prospective observational study
}

\author{
Lisa K Kuramoto, ${ }^{1}$ Boris G Sobolev, ${ }^{1,2}$ Penelope M A Brasher, ${ }^{1}$ Michael W Tang, ${ }^{1}$ \\ Jacquelyn J Cragg (iD ${ }^{3,4}$
}

To cite: Kuramoto LK, Sobolev BG, Brasher PMA, et al. Constructing treatment episodes from concomitant medication logs: a prospective observational study. BMJ Open 2020;10:e034305. doi:10.1136/ bmjopen-2019-034305

- Prepublication history for this paper is available online. To view these files, please visit the journal online (http://dx.doi. org/10.1136/bmjopen-2019034305).

Received 13 September 2019 Revised 23 January 2020 Accepted 10 February 2020

Check for updates

(C) Author(s) (or their employer(s)) 2020. Re-use permitted under CC BY-NC. No commercial re-use. See rights and permissions. Published by BMJ.

${ }^{1}$ Centre for Clinical Epidemiology and Evaluation, Vancouver Coastal Health Research Institute, The University of British Columbia, Vancouver, British Columbia, Canada

${ }^{2}$ School of Population and Public Health, The University of British Columbia, Vancouver, British Columbia, Canada

${ }^{3}$ Faculty of Pharmaceutical Sciences, The University of British Columbia, Vancouver, British Columbia, Canada ${ }^{4}$ International Collaboration on Repair Discoveries (ICORD), The University of British Columbia, Vancouver, British Columbia, Canada

Correspondence to

Dr Jacquelyn J Cragg;

jacquelyn.cragg@icord.org

\section{ABSTRACT}

Objectives To describe an approach using concomitant medication log records for the construction of treatment episodes. Concomitant medication log records are routinely collected in clinical studies. Unlike prescription and dispensing records, concomitant medication logs collect utilisation data. Logs can provide information about drug safety and drug repurposing.

Design A prospective multicentre, multicohort observational study.

Setting Twenty-one clinical sites in the USA, Europe, Israel and Australia.

Participants 415 subjects from the de novo cohort of the Parkinson's Progression Markers Initiative.

Methods We construct treatment episodes of concomitant medication use. The proposed approach treats temporal gaps as a stoppage of medication and temporal overlaps as simultaneous use or changes in dose. Log records with no temporal gaps were combined into a single treatment episode.

Results 5723 concomitant medication log records were used to construct 3655 treatment episodes for 65 medications. There were 405 temporal gaps representing a stoppage of medication; 985 temporal overlaps representing simultaneous regimens of the same medication and 2696 temporal overlaps representing a change in dose regimen. The median episode duration was 37 months (IQ interval: 11-73 months).

Conclusions The proposed approach for constructing treatment episodes offers a method of estimating duration and dose of treatment from concomitant medication log records. The accompanying recommendations guide log data collection to improve their quality for drug safety and drug repurposing.

\section{INTRODUCTION}

Concomitant medication $\log$ records are routinely collected in clinical studies. Their collection is important for establishing safety of new investigational drugs. For example, medications may interact with study interventions or interact with contrast media used in imaging procedures. Regulatory agencies, such as Health Canada and the US Food and

\section{Strengths and limitations of this study}

A large, observational study prospectively capturing information on medication use in an internationally representative cohort of individuals with Parkinson's disease.

Offers an approach for using concomitant medication log records for drug surveillance and drug repurposing studies.

- Assumes concomitant medication logs accurately capture subjects' medication use.

Drug Administration, require that concomitant medications be recorded during clinical trials and prospective cohort studies. ${ }^{1}$ Concomitant medication records can add to the drug safety profiles of commonly used, approved drugs. For example, concomitant medication records from a completed clinical trial were used to test whether a common antispasticity medication interfered with motor neurological recovery. ${ }^{2}$ Concomitant medication records can also identify targets for drug repurposing. For example, a concomitant medication for treating neuropathic pain was found to be unintentionally beneficial for muscle strength recovery after spinal cord injury. ${ }^{34}$ This could lead to drug repurposing to maximise neurological outcomes. ${ }^{5}$

Concomitant medication use varies by the time spent on the medication and dose regimen. These variations are captured by treatment episodes, the events from the start to the end of the administration of a medication. ${ }^{6}$ Specifically, construction of treatment episodes identifies events of medication use belonging to the same treatment, and identifies dose changes during the episode. Construction of treatment episodes plays a key role in calculating measures of exposure which are used in statistical analyses for estimating medication effects. ${ }^{7-10}$ Some measures of exposure, which can be derived from episodes, include an indicator of current use, 
episode duration or episode dose. ${ }^{11}$ Episode duration is the time exposed to the medication. Episode dose is the total daily dosage over the course of the episode.

Different methods and assumptions used in episode construction influence measures of exposure and effect. ${ }^{6}$ Underestimating treatment effects may result in missed opportunities for therapeutic development, while overestimating treatment effects may result in wasted resources. While the pharmacoepidemiology literature describes treatment episode construction, ${ }^{671213}$ the data used in these cases are information about medications prescribed or dispensed as opposed to medication utilisation. ${ }^{14} 15$ Our objective was to describe an approach using concomitant medication $\log$ records for the construction of treatment episodes. We also provide recommendations for collecting concomitant medication data to improve their quality and use in drug safety and drug repurposing strategies.

\section{METHODS}

\section{Data source}

Data were obtained from the Parkinson's Progression Markers Initiative (PPMI) database. The PPMI is a comprehensive prospective observational study designed to improve understanding of disease aetiology and progression. ${ }^{16} 17$ The PPMI database is composed of multiple cohorts including recently diagnosed Parkinson's disease (PD) subjects and healthy participants followed longitudinally for clinical, imaging, behavioural and biospecimen assessments, using standardised data acquisition protocols at 21 clinical sites in the USA, Europe, Israel and Australia. Anonymised study data are publicly available through the PPMI website (www.ppmi-info.org). We downloaded the PPMI data files on 6 April 2018. ${ }^{18}$

\section{Patient and public involvement}

Patients or the public were not involved in the design, or conduct, or reporting, or dissemination of our research.

\section{PPMI concomitant medication log}

The collection of concomitant medications in PPMI is done through a standardised case report form (CRF) ${ }^{16} \mathrm{In}$ PPMI, medications taken at Screening Visit (the visit used to screen for study eligibility) are entered into the log. At subsequent visits, new medications and changes/discontinuation of previously listed medications are recorded. The following instructions are provided on the CRF:

Enter all medications taken at Screening Visit. At subsequent visits record new meds, and changes/discontinuation of previously listed meds. Changes in total daily dose or route require a new line. Row: enter 1 , 2, 3, etc. Medication: Record generic name; if unknown, enter brand name. For multiple ingredient medications, indicate strength if possible, for example, carbidopa/levodopa 25/100. Dose: Record dose for each administration. Date: Please specify if the Start and Stop dates are ACTUAL or ESTIMATED. If the exact date is unknown, please enter your best reasonable estimate of the date and specify which part(s) are estimated. Ongoing: Answer yes if medication is still being taken at end of study. Indication: Reason for use, not drug category.

Data from this CRF are entered into the PPMI publicly accessible concomitant medication log data file. This data file includes medication name, dose, dose units, frequency, route, start/stop date (or ongoing), indication, and whether the medication is indicated for PD (table 1). Only month and year of the start and stop dates are publicly accessible. The data file also contains derived medication variables, including the WHO drug code. ${ }^{19}$ The WHO drug code has three parts: drug record number (RECNO), sequence number 1 (SEQNO1) and sequence number 2 (SEQNO2). RECNO uniquely identifies active moieties.

We included log records for RECNOs that a minimum of 20 de novo PD subjects were taking at the screening visit or during the follow-up. This number was an arbitrary cut-off to identify common medications. We also excluded the 'all other non-therapeutic products' (ie, RECNO=900475) due to a lack of known biological activity. Examples of this included products such as blueberry extract, probiotics and Neuroplex.

\section{Episode construction}

The proposed approach is founded on the assumption that concomitant medication logs capture the patients' actual medication use. We assume temporal gaps represent a stoppage of medication; temporal overlaps of more than one time unit (eg, month) represent simultaneous regimens of the same medication; and temporal overlaps of one time unit represent a change in dose regimen. Temporal gaps are the elapsed time between the end of one $\log$ record and the start of the subsequent log record. Temporal overlaps are the time during which a log record starts before a previous log record ends. Since only the year and month of the medication start and stop dates were accessible, we distinguished between simultaneous regimens and changes in dose regimens to not overestimate total daily dose. $\log$ records with the same RECNO and no temporal gaps were combined into a single treatment episode.

Log records with a medication frequency of 'as needed' (eg, 'PRN', 'OCCASIONAL', 'ON DEMAND', 'QS', or 'SOS') did not contribute to exposure because we could not confirm medication utilisation. When the log record had a vague unit (eg, tablet or capsule) the daily dose could not be computed; however, the record still contributed to episode duration.

\section{Episode duration}

We identified the start and end dates of medication use for each $\log$ record. If there was no start date or if the dates were non-chronological, we marked the log record as incomplete. If there was no stop date, we imputed with the date of the last concomitant medication review as the 
Table 1 Data dictionary for concomitant medication log data file from the Parkinson's Progression Markers Initiative study

\begin{tabular}{|c|c|c|}
\hline Variable & Description & Type \\
\hline REC_ID & Record ID & No \\
\hline F_STATUS & $\begin{array}{l}\text { Data status: verified, } \\
\text { secured or locked }\end{array}$ & Char \\
\hline CNO & Centre no & Char \\
\hline PATNO & Patient no & Char \\
\hline EVENT_ID & Event name & Char \\
\hline PAG_NAME & Page name & Char \\
\hline INVSTAFF & Investigator staff code & Char \\
\hline CMSEQ & Row \# & No \\
\hline CMTRT & Medication & Char \\
\hline CMDOSE & Dose & No \\
\hline CMDOSU & Units & Char \\
\hline CMDOSFRQ & Frequency & Char \\
\hline ROUTE & Route & Char \\
\hline STARTDT & Start date & Date \\
\hline STARTEST & Start date estimation & Char \\
\hline STOPDT & Stop date & Date \\
\hline STOPEST & Stop date estimation & Char \\
\hline ONGOING & Ongoing & Char \\
\hline CMINDC & Indication & Char \\
\hline DISMED & PD med? & Char \\
\hline TOTDDOSE & Total daily dose & No \\
\hline RECNO & WHO RECNO & Char \\
\hline SEQNO1 & WHO SEQNO1 & Char \\
\hline SEQNO2 & WHO SEQNO2 & Char \\
\hline WHODRUG & WHO drug name & Char \\
\hline EXCLMED & Exclusionary med flag & Char \\
\hline LEDD & $\begin{array}{l}\text { LEDD calculation for PD } \\
\text { medication }\end{array}$ & No \\
\hline ORIG_ENTRY & Date of original data entry & Date \\
\hline LAST_UPDATE & $\begin{array}{l}\text { Date of most recent } \\
\text { update to record }\end{array}$ & Date \\
\hline QUERY & $\begin{array}{l}\text { Any open/pending queries } \\
\text { on this record }\end{array}$ & No \\
\hline SITE_APRV & $\begin{array}{l}\text { Date site approved the } \\
\text { data }\end{array}$ & Date \\
\hline
\end{tabular}

LEDD, Levodopa equivalent daily dose; PD, Parkinson's disease; RECNO, record number; SEQNO1, sequence number 1; SEQNO2, sequence number 2.

PPMI protocol instructed to leave this field blank until either the subject ended participation or the study was over.

Among log records belonging to the same treatment episode, the episode start date was the start date of the earliest log record and the episode end date was the stop date of the latest log record. The episode duration was computed as the number of months between the episode start and end dates.

\section{Episode dose}

We calculated the daily dose from each log record. First, we determined the multiplicative factor, representing how often the reported dose is taken per day. For example, 'BID' means twice a day, so the multiplicative factor is 2. Next, we converted doses to a common unit (eg, mg). The daily dose is the product of the reported dose in common units and the multiplicative factor based on the frequency.

We calculated the total daily dose among log records belonging to the same treatment episode. The total daily dose at a time with simultaneous regimens was the sum of the daily doses of log records containing the time point (figure 1). The total daily dose at a time with changes in dose regimen was the average of the doses (figure 1). If there was only one log record constructing an episode, then the total daily dose was equal to the daily dose. The total daily dose and any changes were computed over the course of the treatment episode.

\section{RESULTS \\ Episode durations}

Of the 423 enrolled de novo PD subjects, 415 had a baseline visit and at least one follow-up visit. Of the 8771 concomitant medication log records, we excluded 2619 $(29.9 \%) \log$ records of medications taken by fewer than 20 PD subjects, $352(4.0 \%)$ records with drug frequencies of 'as needed' and $77(0.9 \%) \log$ records for 'all other non-therapeutic products' (ie, RECNO=9 00475). The remaining $5723 \log$ records were used to construct treatment episodes for 65 medications among the 415 PD subjects. Two-fifths of the log records were incomplete: 3 $(0.05 \%)$ had no start date, $2328(40.7 \%)$ had no stop date (so were imputed) and $2(0.03 \%)$ had non-chronological start and stop dates. For example, REC_ID 282665101 recorded that PATNO 3606 had no start date or stop date for a vitamin B complex. REC_ID 226846701 recorded that PATNO 3400 started Lexapro in February 2010, but had no stop date. REC_ID 538226701 had a start date on February 2013 and a stop date on March 2012; and REC_ ID 413205901 had a start date on September 2013 and stop date on August 2013. These log records, as with records with data entry errors (eg, REC_ID 667793101 with a stop date of ' $03 / 0218$ '), were considered incomplete.

About half of the log records were combined to form treatment episodes: $543(9.5 \%)$ log records had identical start and stop dates; 132 (2.3\%) records started and stopped a medication regimen before stopping the previous regimen; and $2260(39.5 \%)$ records started another regimen of the same medication before stopping the previous regimen. The remaining log records each corresponded to a single treatment episode. There were 405 temporal gaps among subjects with multiple log 


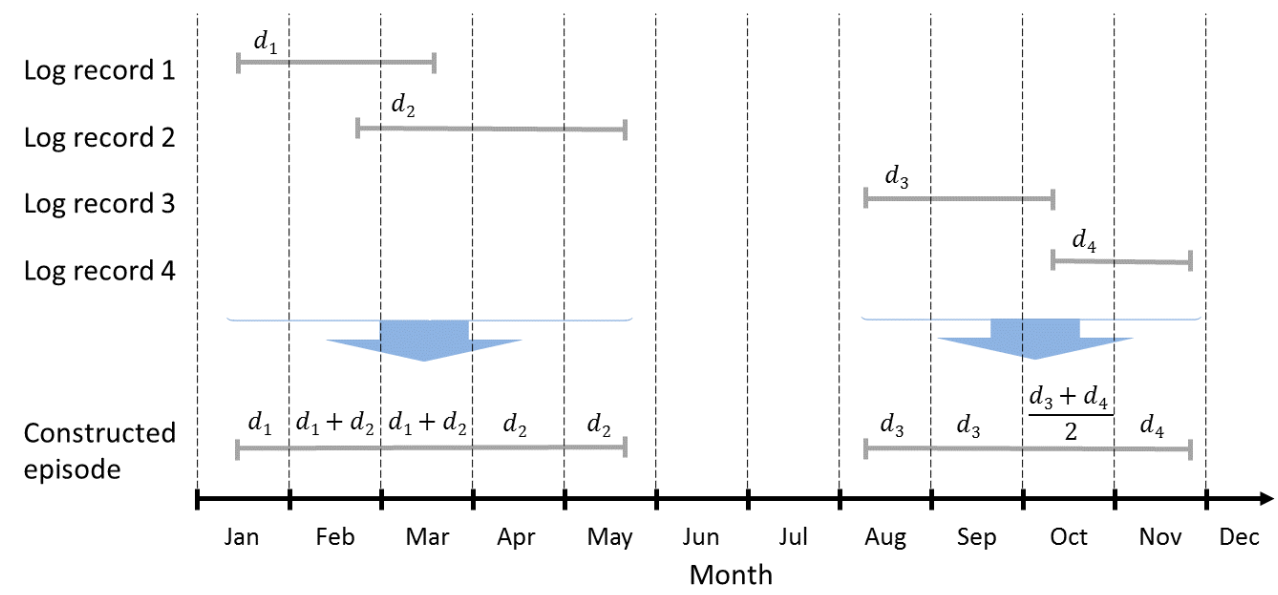

Figure 1 Treatment episodes constructed from overlapping log records for the same medication. The log records have varying durations and different daily doses with common units, $d_{1}, d_{2}, d_{3}$ and $d_{4}$. Log records 1 and 2 belong to a treatment episode with simultaneous regimens of the same medication. Log records 3 and 4 belong to another treatment episode with a change in dose regimen. Constructed episodes show total daily dose for each month.

records for a given medication. The gaps had a median duration of 8 months (IQ interval (IQI): 1-14 months).

The 415 de novo PD subjects had 60 months median follow-up time (IQI: 54-86 months). We constructed 3655 treatment episodes for 65 medications among these subjects. The median episode duration was 37 months (IQI: 11-73 months).

\section{Episode doses}

There was significant variation in units and frequencies: 66 different units and 136 different frequencies among the $5723 \log$ records. For example, for RECNO 3686, some of the units were reported as TAB, TABS, CAP or CAPS, and some of the frequencies were reported as $4 \mathrm{X}$ DAILY, $4 \mathrm{X}$ QD, 4X, 4X/D, 4XD, 4XDAY, 4XQD or 4XS/ DAY. We revised the reported units to be consistent across records. For example, G, GM, GR, GRAM, ',G', GRAMS were recoded as GRAM. We determined the multiplicative factor for computing daily doses. For example, 6 PER DAY, 6X A DAY, 6/DAY, 6X DAY, 6X/DAY, 6XD, 6XS/ DAY, SIX DAILY had a multiplicative factor of 6 . In 8 $(0.1 \%)$ and $95(1.7 \%)$ of the log records, the values for units or frequencies, respectively, were vague.

We calculated the total daily dose during a treatment episode by accounting for temporal gaps and overlaps. There were 405 temporal gaps representing a stoppage of the medication; 985 temporal overlaps of more than one time unit (eg, month) representing simultaneous regimens of the same medication; and 2696 temporal overlaps of one time unit representing a change in dose regimen.

\section{Example: gabapentin}

To illustrate these steps with respect to a single medication, we provide here an example with gabapentin (primarily indicated for neuropathic pain). Among the 415 de novo PD subjects, there were $49 \log$ records of gabapentin use among 30 subjects. For each record of gabapentin, we computed the daily dose. First, we identified $\log$ records of interest (those with RECNO=10030).
Second, we determined the multiplicative factors for calculating daily dose. Third, we converted all units to mg. Finally, we computed the daily dose for gabapentin. For example, PATNO 3625 has a dose of 300 with units MG and frequency BID. Therefore, the daily dose is $300 \mathrm{MG} *$ $2=600 \mathrm{MG}$. One log record indicated that the subject was on the medication 'as needed' and another log record reported units as tablet (ie, 'TAB'), so the records did not contribute to episode duration or dose. Among the remaining gabapentin log records, the median daily dose was $600 \mathrm{mg}$, and ranged from 100 to $3600 \mathrm{mg}$ per day.

We constructed 37 episodes of gabapentin use among subjects. Each user of gabapentin had one or two episodes during the study period. About three-quarters (23 of 30) of these subjects had episodes of gabapentin use starting after the baseline visit. The median cumulative months of exposure was 16 months (IQI: 7-29 months). The median cumulative total daily dose was $156000 \mathrm{mg}$ (IQI: 42600-667800 mg).

\section{DISCUSSION}

We described an approach for the construction of treatment episodes from concomitant medication logs to estimate duration and dose. The approach treats temporal gaps as a stoppage of medication and temporal overlaps as simultaneous use or changes in dose. Log records with no temporal gaps were combined into a single treatment episode.

Construction of treatment episodes using concomitant medication $\log$ data differs from construction using drug prescribing and dispensing data (table 2) ${ }^{671213}$ Approaches for constructing episodes from prescription or dispensing records are used to infer medication use, whereas the proposed approach is based on actual medication use. Data from prescription and dispensing databases are typically less comprehensive (eg, may not include a route of administration, generally do not capture non-prescription drugs, 
sometimes limited to a specific setting such as a single hospital or pharmacy, often restricted in terms of number of years captured, generally do not include drug indication). Second, in concomitant medication logs, temporal gaps in medication use identify when the subject was not actually taking the drug. This is in contrast to prescription and dispensing data, where gaps of predefined length are filled when constructing episodes to account for assumed medication use during those gaps. In some cases, patientreported medication use is the reference standard for prescribing/dispensing records, ${ }^{20}$ supporting treating gaps as a stoppage of the medication. Lastly, in concomitant medication logs, temporal overlaps identify when the subject is administering simultaneous regimens of the same medication. This is in contrast to prescription and dispensing data, where the overlapping time is used to extend the duration of the episode.

\section{Recommendations}

We submit that concomitant medication logs can be used as a surveillance tool for drug safety and drug repurposing studies. For example, the cumulative exposure

Table 2 Approaches for constructing treatment episodes for medication utilisation

\begin{tabular}{|c|c|c|c|c|}
\hline Issue & Proposed approach & Alternative approaches & $\begin{array}{l}\text { Pros of proposed } \\
\text { approach }\end{array}$ & $\begin{array}{l}\text { Cons of proposed } \\
\text { approach }\end{array}$ \\
\hline Data source & $\begin{array}{l}\text { Concomitant } \\
\text { medication log }\end{array}$ & $\begin{array}{l}\text { - Prescription data } \\
\text { - Drug dispensing data }\end{array}$ & $\begin{array}{l}\text { Aims to capture } \\
\text { drug utilisation, as } \\
\text { opposed to prescribing } \\
\text { or dispensing of } \\
\text { medications } \\
\text { More comprehensive } \\
\text { (eg, captures route of } \\
\text { administration, non- } \\
\text { prescription drugs) }\end{array}$ & $\begin{array}{l}\text { Relies on accurate } \\
\text { reporting of medication } \\
\text { use } \\
\text { Not subjected to same } \\
\text { level of data quality } \\
\text { checks as primary data }\end{array}$ \\
\hline $\begin{array}{l}\text { Temporal } \\
\text { gaps }\end{array}$ & $\begin{array}{l}\text { Assumes gaps } \\
\text { represent a stoppage in } \\
\text { medication use } \\
\text { Do not fill gaps } \\
\text { Records with gaps } \\
\text { between them come } \\
\text { from different episodes. }\end{array}$ & $\begin{array}{l}\text { Assumes gaps could } \\
\text { represent medication use } \\
\text { Fills gaps of predefined } \\
\text { length } \\
\text { Records with gaps within } \\
\text { the predefined length } \\
\text { come from the same } \\
\text { episode. }\end{array}$ & $\begin{array}{l}\text { Objectively identifies } \\
\text { when medication is not } \\
\text { in use } \\
\text { Predefined gap length } \\
\text { is not medication } \\
\text { dependent }\end{array}$ & $\begin{array}{l}\text { Short gaps may be } \\
\text { misclassified as no } \\
\text { medication use }\end{array}$ \\
\hline $\begin{array}{l}\text { Temporal } \\
\text { overlap }\end{array}$ & $\begin{array}{l}\text { Assumes overlaps } \\
\text { represent simultaneous } \\
\text { or change in medication } \\
\text { regimen } \\
\text { Total daily dose is } \\
\text { computed as a sum or } \\
\text { average over records } \\
\text { Overlapping records } \\
\text { come from the same } \\
\text { episode }\end{array}$ & $\begin{array}{l}\text { Assumes overlaps } \\
\text { represent re-filling } \\
\text { prescriptions early } \\
\text { Overlapping time added } \\
\text { to episode duration or } \\
\text { ignored } \\
\text { Overlapping records } \\
\text { come from the same } \\
\text { episode }\end{array}$ & $\begin{array}{l}\text { Objectively identifies } \\
\text { episode end date }\end{array}$ & $\begin{array}{l}\text { Potential } \\
\text { overestimation of total } \\
\text { daily dose, if overlap } \\
\text { erroneously represents } \\
\text { double reporting }\end{array}$ \\
\hline $\begin{array}{l}\text { Medication } \\
\text { start and } \\
\text { stop dates }\end{array}$ & $\begin{array}{l}\text { Assumes record } \\
\text { captures actual } \\
\text { medication start and } \\
\text { stop dates } \\
\text { Impute missing stop } \\
\text { dates with date of last } \\
\text { medication review } \\
\text { Mark records with no } \\
\text { start date as incomplete }\end{array}$ & $\begin{array}{l}\text { Assumes medication } \\
\text { start date is the } \\
\text { prescription or } \\
\text { dispensing date } \\
\text { Infers medication stop } \\
\text { date from prescription } \\
\text { order } \\
\text { Mark records with no } \\
\text { start/stop dates as } \\
\text { incomplete }\end{array}$ & $\begin{array}{l}\text { Uses actual medication } \\
\text { start and stop dates }\end{array}$ & $\begin{array}{l}\text { Potential } \\
\text { overestimation of } \\
\text { episode duration, if } \\
\text { imputing stop date }\end{array}$ \\
\hline
\end{tabular}


to gabapentin for pain management, computed using the proposed approach, could, in future studies, be correlated with motor disease progression to see if the drug is detrimental (ie, drug safety) or beneficial (ie, candidate for repurposing) in PD. In addition, both sponsors and regulatory agencies may inspect these types of logs over a study lifespan to ensure compliance with trial design and patient adherence. ${ }^{21}$

Applying the proposed approach to the data from the PPMI concomitant medication log yielded recommendations for data collection and data quality checks for future prospective studies (box 1). When collecting data for a concomitant medication log, we recommend: (1) quality checks for valid start dates (eg, no data entry errors); (2) quality checks for valid stop dates (eg, start and stop dates are chronologically ordered); (3) record medication start and stop dates with day, month, and year (ie, complete dates for precise account of medication use); (4) quality checks for valid dose (eg, dose is within the recommended range); (5) quality checks for valid unit (eg, no vague units like 'tablet'); (6) quality checks for valid frequency (eg, confirm the use of 'as needed' medication with the subject); (7) quality checks for overlapping log records with the same medication, dose and frequency; (8) continuous recording of concomitant medication status (so start and stop dates are more accurate), by e-diaries, for example; (9) electronic log records to prevent users from creating another log record for the same medication, if a log record for the medication with no stop date exists (ie, is there a need to have two 'active' records for the same medication') and (10) use standardised drug/ indication systems, such as WHO system.

\section{Study limitations}

Our study has a few limitations. First, the proposed approach assumes that the concomitant medication log data accurately capture the subjects' medication use. The PPMI data indicate whether start and stop dates were actual or estimated. The month of the start and stop dates was estimated in $39 \%$ and $21 \%$ of records, respectively. While patient report has been used as a standard in prior studies, the validity may differ with respect to different medications.

Second, the approach does not specify how to handle errors in medication start dates, stop dates or doses. These errors could result in underestimating or overestimating

Box 1 Recommendations for collecting concomitant medication log data

Check validity of start and stop dates.

- Record start and stop dates with year, month and day.

- Check validity of dose, unit and frequency.

- Check validity of overlapping log records.

- Record medication status continuously.

- Electronic logs to prevent invalid entries.

Use standardised drug and indication systems. episode durations or doses. For example, the approach assumes that temporal overlaps represent simultaneous use; however, it could be an error of double reporting. In this case, the approach would overestimate the episode dose, but episode duration could be unaltered. In a sensitivity analysis, we reconstructed episodes assuming that temporal overlaps represented an error in double reporting. We retained the overlap from the most recent record. For gabapentin, the cumulative months of exposure and the cumulative total daily dose had medians that remained the same, but the IQI became narrower for the latter (42600-580500 mg).

Third, we assumed no medication use when the medication frequency was 'as needed' which could underestimate the medication duration and dose.

The advantage of our study is that we had longitudinal medication information in an internationally representative cohort of individuals with PD.

\section{CONCLUSION}

The proposed approach to constructing treatment episodes offers a method of estimating duration and dose of medication treatment using data from concomitant medication logs. The accompanying recommendations guide log data collection in clinical studies to improve their quality and use in drug safety and drug repurposing strategies.

Acknowledgements PPMI—a public-private partnership-is funded by the Michael J. Fox Foundation for Parkinson's Research and funding partners, including Abbvie, Allergan, Avid Radiopharmaceuticals, Biogen, BioLegend, Bristol-Myers Squibb, Celgene, Denali, GE Healthcare, Genentech, GlaxoSmithKline, Lilly, Lundbeck, Merck, Meso Scale Discovery, Pfizer, Piramal, Prevail Therapeutics, Roche, Sanofi Genzyme, Servier, Takeda, Teva, UCB, Verily and Voyager Therapeutics.

Contributors All authors contributed to and approved the final manuscript. JC conceived the work and obtained study funding; LK and MT analysed the data; LK, BS, PB, MT and JC interpreted the data for the work; LK and JC drafted the manuscript; and LK, BS, PB, MT and JC revised the manuscript for important intellectual content.

Funding This work was supported by the Michael J. Fox Foundation for Parkinson's research (grant number 14834).

Competing interests None declared.

Patient consent for publication Not required.

Ethics approval This study, which involved publicly available data, was exempt from ethics review according to National policy.

Provenance and peer review Not commissioned; externally peer reviewed.

Data availability statement Data are available on reasonable request. Anonymised study data are publicly available through the PPMI website (www. ppmi-info.org). Investigators seeking access to PPMI data must submit an online application, which requires signing the Data Use Agreement and compliance with the study Publications Policy.

Open access This is an open access article distributed in accordance with the Creative Commons Attribution Non Commercial (CC BY-NC 4.0) license, which permits others to distribute, remix, adapt, build upon this work non-commercially, and license their derivative works on different terms, provided the original work is properly cited, appropriate credit is given, any changes made indicated, and the use is non-commercial. See: http://creativecommons.org/licenses/by-nc/4.0/.

ORCID iD

Jacquelyn J Cragg http://orcid.org/0000-0002-6284-938X 


\section{REFERENCES}

1 U.S. Department of Health and Human Services, Food and Drug Administration C for DE and R (CDER). Guidance for industry. drug interaction Studies- study design, data analysis, implications for dosing, and labeling recommendations. Guid Doc 2012.

2 Cragg JJ, Tong B, Jutzeler CR, et al. A longitudinal study of the neurologic safety of acute baclofen use after spinal cord injury. Neurotherapeutics 2019;16:858-67.

3 Cragg JJ, Haefeli J, Jutzeler CR, et al. Effects of pain and pain management on motor recovery of spinal cord-injured patients: a longitudinal study. Neurorehabil Neural Repair 2016;30:753-61.

4 Warner FM, Cragg JJ, Jutzeler CR, et al. Early administration of Gabapentinoids improves motor recovery after human spinal cord injury. Cell Rep 2017;18:1614-8.

5 Ashburn TT, Thor KB. Drug repositioning: identifying and developing new uses for existing drugs. Nat Rev Drug Discov 2004;3:673-83.

6 Gardarsdottir H, Souverein PC, Egberts TCG, et al. Construction of drug treatment episodes from drug-dispensing histories is influenced by the gap length. J Clin Epidemiol 2010;63:422-7.

7 Pazzagli L, Linder M, Zhang M, et al. Methods for time-varying exposure related problems in pharmacoepidemiology: an overview. Pharmacoepidemiol Drug Saf 2018;27:148-60.

8 Sylvestre M-P, Abrahamowicz M. Flexible modeling of the cumulative effects of time-dependent exposures on the hazard. Stat Med 2009;28:3437-53.

9 Abrahamowicz M, Beauchamp M-E, Sylvestre M-P. Comparison of alternative models for linking drug exposure with adverse effects. Stat Med 2012;31:1014-30.

10 Thomas DC. Models for exposure-time-response relationships with applications to cancer epidemiology. Annu Rev Public Health 1988;9:451-82.
11 Abrahamowicz M, Bartlett G, Tamblyn R, et al. Modeling cumulative dose and exposure duration provided insights regarding the associations between benzodiazepines and injuries. $J$ Clin Epidemiol 2006;59:393-403.

12 Mantel-Teeuwisse AK, Klungel OH, Verschuren WM, et al. Comparison of different methods to estimate prevalence of drug use by using pharmacy records. J Clin Epidemiol 2001;54:1181-6.

13 Khotimah PH, Sugiyama Y, Yoshikawa M, et al. Medication episode construction framework for retrospective database analyses of patients with chronic diseases. IEEE J Biomed Heal Inform 2018;22:1949-59.

14 McKenzie DA, Semradek J, McFarland BH, et al. The validity of Medicaid pharmacy claims for estimating drug use among elderly nursing home residents: the Oregon experience. J Clin Epidemiol 2000;53:1248-57.

15 Løkkegaard EL, Johnsen SP, Heitmann BL, et al. The validity of selfreported use of hormone replacement therapy among Danish nurses. Acta Obstet Gynecol Scand 2004;83:476-81.

16 Parkinson Progression Marker Initiative. The Parkinson progression marker initiative (PPMI). Prog Neurobiol 2011;95:629-35.

17 Latourelle JC, Beste MT, Hadzi TC, et al. Large-Scale identification of clinical and genetic predictors of motor progression in patients with newly diagnosed Parkinson's disease: a longitudinal cohort study and validation. Lancet Neurol 2017;16:908-16.

18 Interagency Advisory Panel on Research Ethics. TCPS 2 (2014) - the latest edition of Tri-Council Policy Statement: Ethical Conduct for Research Involving Humans. Panel Res Ethics 2014.

19 Nahler G. Who collaborating centre for international drug monitoring Dict Pharm Med 2010.

20 Ray WA, Murray KT, Hall K, et al. Azithromycin and the risk of cardiovascular death. N Engl J Med 2012;366:1881-90.

21 ICH E3. Structure and content of clinical study reports. Good Clin Pract J 2001. 